\title{
R script
}

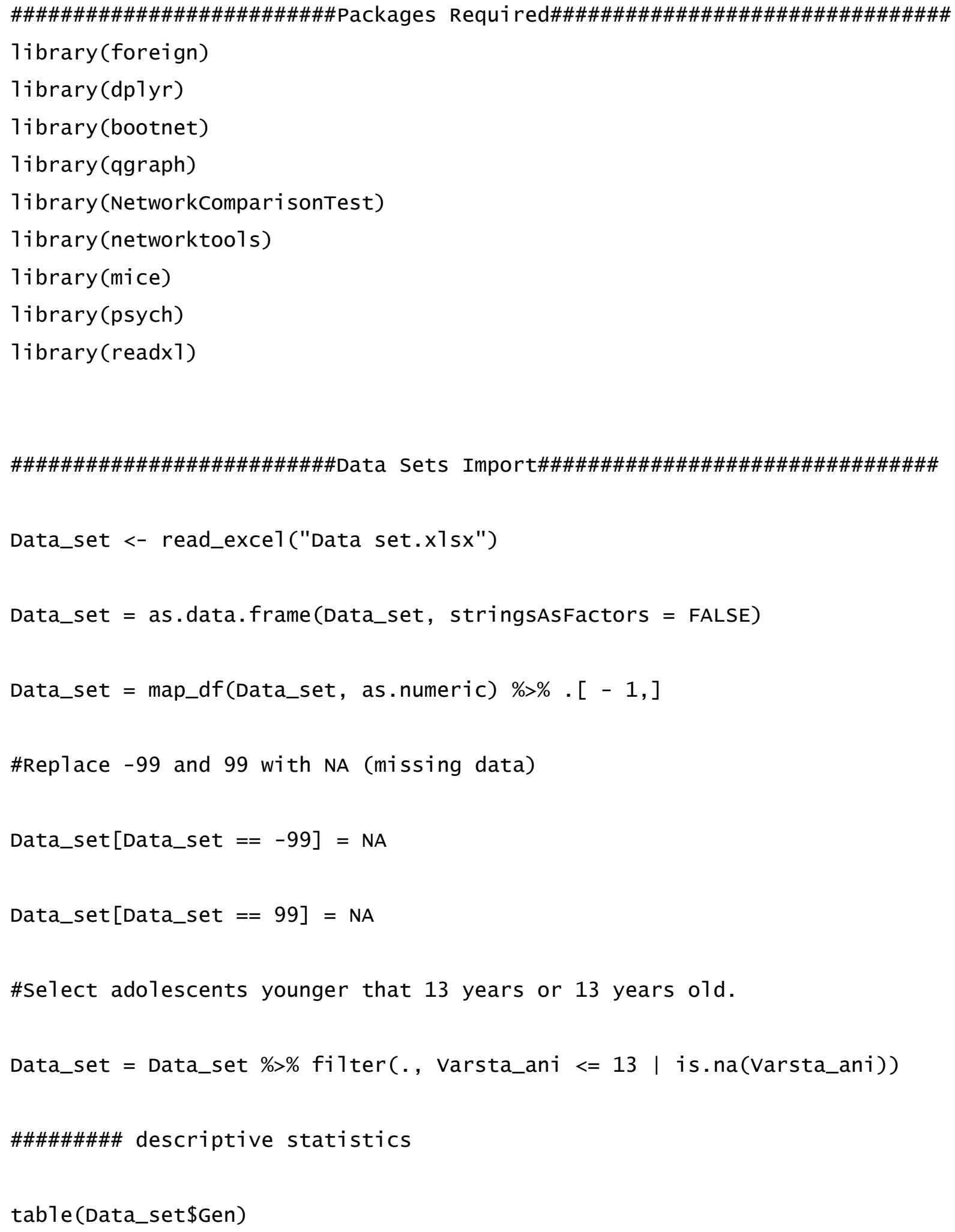




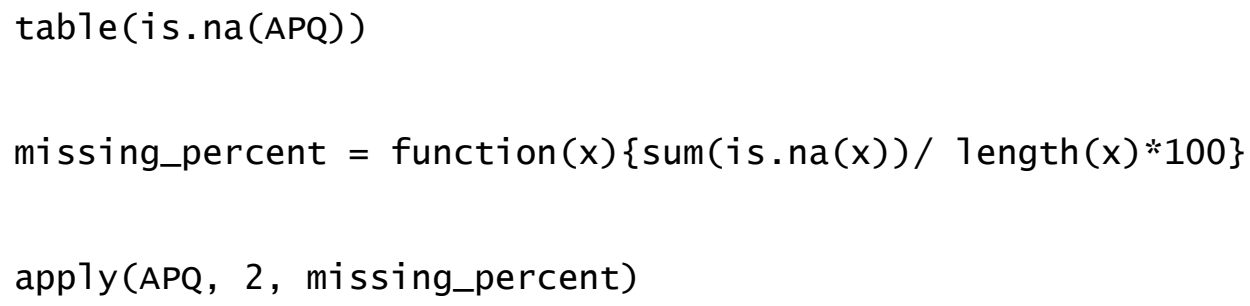




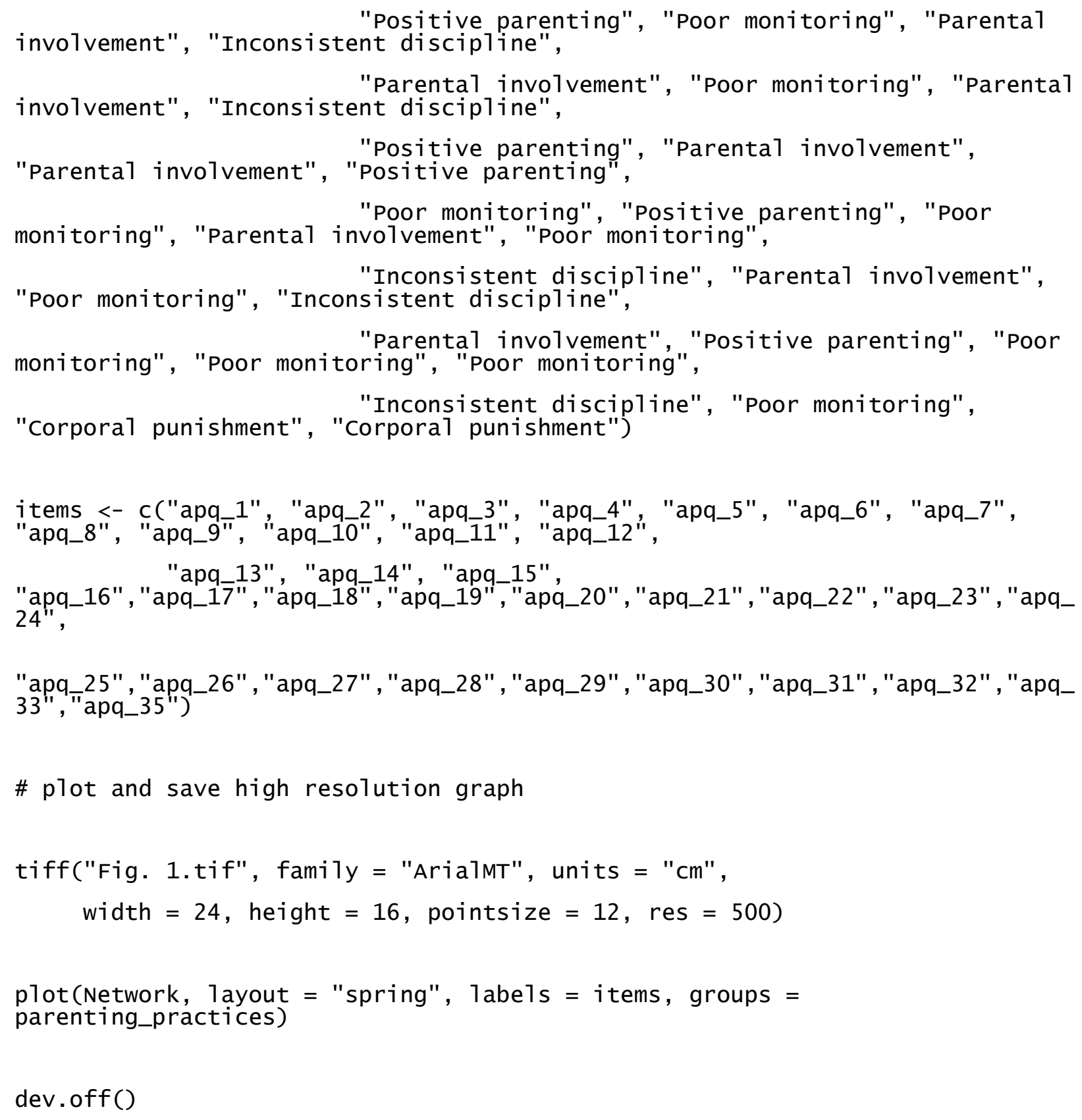


dev. off()

\#\#\#\#\#\#\#\#\#\#\#\#\#\#\#\#\#1ge-Weights Accuracy\#\#\#\#\#\#\#\#\#\#\#\#\#\#\#\#\#\#\#\#\#\#\#\#

boot0 $<-$ bootnet (Network, nBoots $=2500$, nCores $=8$ )

tiff("Fig. S1.tif", fami1y = "Aria1MT", units = "cm", width $=30$, height $=16$, pointsize $=12$, res $=230$ )

plot (boot0, 1abels = FALSE, order = "sample")

dev.off()

\#\#\#\#\#\#\#\#\#\#\#\#\#\#\#The Stability of Centrality Indices\#\#\#\#\#\#\#\#\#\#\#\#\#\#\#\#\#

boot2 <- bootnet (Network, nBoots $=2500$, type = "case", nCores $=8$, statistics = c("strength", "closeness", "betweenness"))

plot (boot2, statistics = c("strength", "closeness", "betweenness")) corstabi1ity(boot2)

\#\#\#\#\#\#\#\#\#\#\#\#\#\#\#\#Significant Difference between Nodes\#\#\#\#\#\#\#\#\#\#\#\#\#\#\#\#

boot1 <- bootnet (Network, nBoots $=2500$, ncores $=8$, statistics $=$ c("strength", "closeness", "betweenness"))

tiff("Fig. S2.tif", family = "Aria1MT", units $=" \mathrm{~cm} "$, width $=30$, height $=16$, pointsize $=12$, res $=230$ )

plot (boot1, 1abe1s = TRUE, order = "sample", statistics = c("strength", "closeness", "betweenness"))

dev.off()

\#\#\#\#\#\#\#\#\#\#\#\#\#\#\#\#\#AQ Bridge Test\#\#\#\#\#\#\#\#\#\#\#\#\#\#\#\#\#

bridge_APQ_network <- getwmat(Network)

qgraph (bridge_APQ_network, groups=parenting_practices, 1ayout="spring")

bridge_centrality <- bridge(bridge_APQ_network, communities=parenting_practices) 


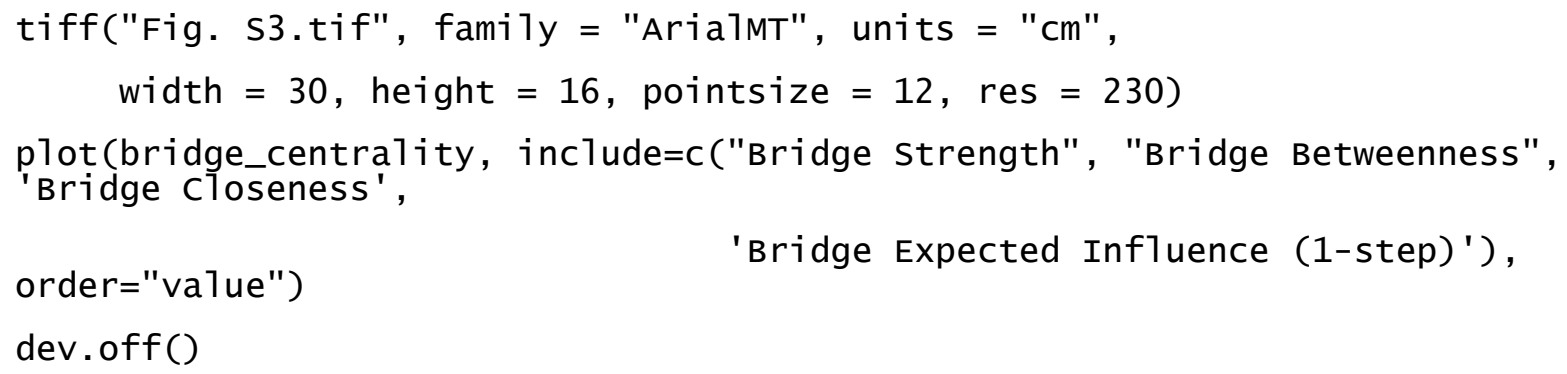


Network_females <- estimatenetwork(APQ_females $[,-c(1,36)]$, corMethod $=$ 'cor_auto', default = "EBICglasso")

plot (Network_females, 1ayout = "spring", 1abe1s = items, groups = parenting_practices)

plot(Network_males, 1ayout = "spring", 1abels = items, groups = parenting_practices)

Females_males_APQ_comparison <- NCT(Network_males, Network_females, it = 1000 , binary. data $=$ FALSE,

abs=TRUE, test. edges=TRUE, paired=FALSE, weighted=TRUE, AND=TRUE, make. positive.definite=TRUE, p.adjust.methods $=$ " c("strength", "expectedInfluence"), nodes="a11", communities=parenting_practices, usecommunities="a11", verbose = TRUE)

plot (Females_males_APQ_comparison, what = 'network')

plot(Females_males_APQ_comparison, what = 'centrality')

plot(Females_males_APQ_comparison, what = 'edge')

plot(Females_males_APQ_comparison, what = 'strength')

print(Females_males_APQ_comparison)

centralityPlot(1ist(Males = Network_males, Females = Network_females), include = C("Strength", "Closeness", "Betweenness", "expected̃nfluence"), 1abe1s = items)

\#\#\#\#\#\#\#\#Screening APQ_YSR Subscales Data\#\#\#\#\#\#\#\#\#\#\#\#\#\#\#\#\#\#\#\#\#\#\# \#\#Compute Total scores on sub scales

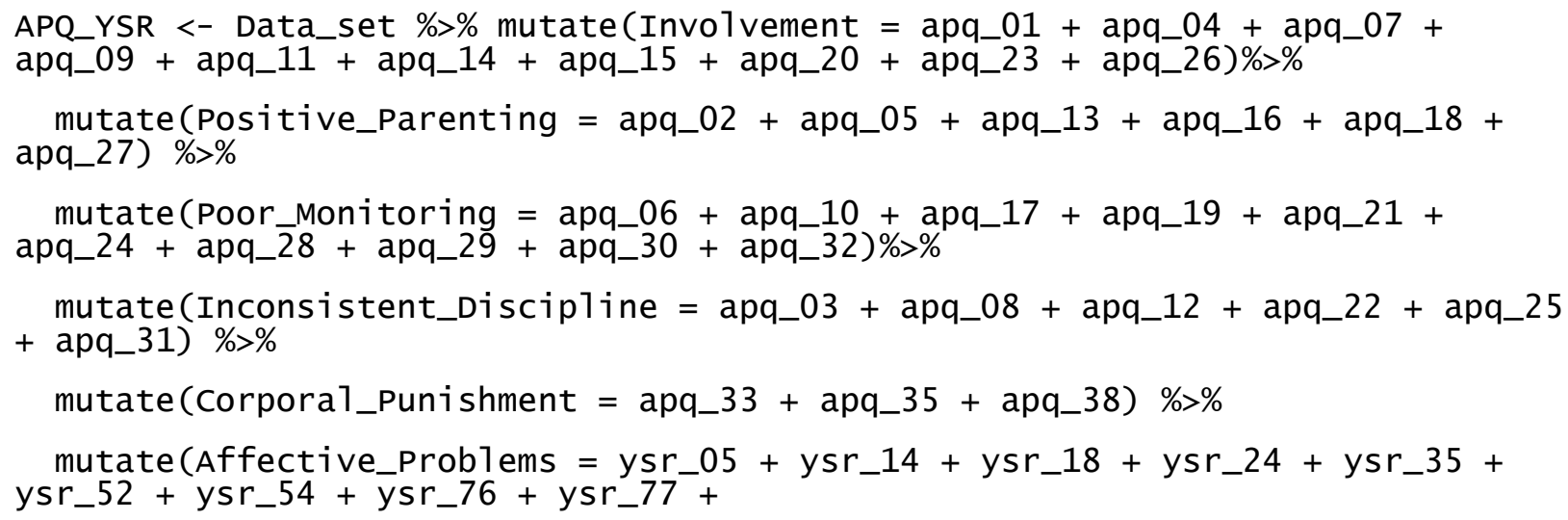




$$
\text { ysr_91 + ysr_100 +ysr_102 +ysr_103) \%>\% }
$$

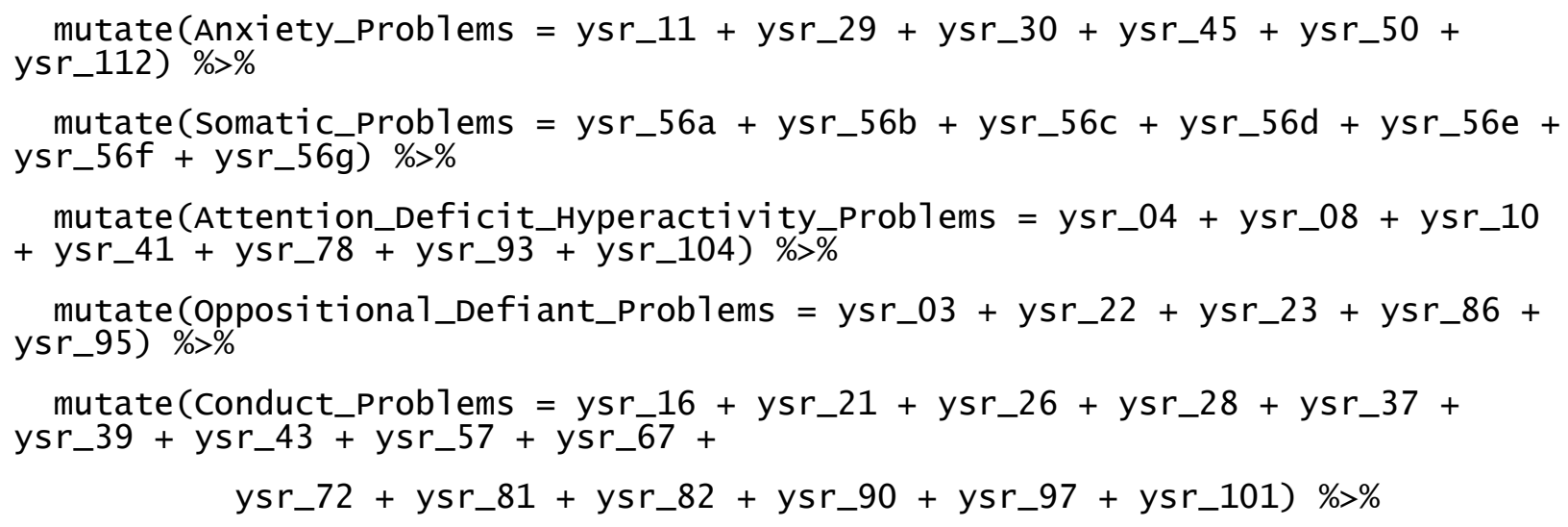

select(Varsta_ani, Gen, Involvement, Positive_Parenting, Poor_Monitoring, Inconsistent_Discipline, Corpora1_Punishment, Affective_Problems, Anxiety_Probiems, Somatic_Problems, Attention_Deficit_Hyperactivity_Problems, oppositiona1_Defiant_Problems, Conduct_Problems)

\# Reliability

\#parental involvement APQ[, c('apq_01', 'apq_04', 'apq_07', 'apq_09', 'apq_11', 'apq_14', 'apq_15' , . \$total

\#positive parenting

APQ[, c('apq_02', 'apq_05', 'apq_13', 'apq_16', 'apq_18', 'apq_27')] \%>\% polychoric() \%>\% . \$rho \%>\% alpha() \%>\% . \$total

\#poor monitoring

APQ [, c ('apq_06', 'apq_10', 'apq_17' , 'apq_19', 'apq_21', 'apq_24', 'apq_28' , 'apq_29', 'apq_30', 'apq_32')] \%>\% polychoric() \%>\% .\$rho \%>\% alpha() \%>\% . \$tota1

\#inconsistent discipline

APQ[, c('apq_03', 'apq_08', 'apq_12', 'apq_22', 'apq_25', 'apq_31')] \%>\% polychoric() \%>\% . \$rho \%>\% alpha() \%>\% . \$total

\#corporal punishment

$\mathrm{APQ}[$, c('apq_33', 'apq_35', 'apq_38')] \%>\% polychoric() \%> . \$rho \%> alpha() $\%>\%$. \$tota1

\# Affective problems 
Data_set [, c('ysr_05', 'ysr_14', 'ysr_18', 'ysr_24', 'ysr_35', 'ysr_52',

'ysr_54', 'ysr_76', 'ysr_77', 'ysr_91', 'ysr_100', 'ysr_102', 'ysr_103')] \%>\% polychoric() \%>\% . \$rho \%>\% alpha() \%>\% . \$total

\# Anxiety problems

Data_set [, c('ysr_11', 'ysr_29', 'ysr_30', 'ysr_45', 'ysr_50', 'ysr_112')]

$\%>\%$ polychoric() \%>\% . \$rho \%>\% alpha() \%>\% . \$total

\# Somatic_Problems

Data_set [, c('ysr_56a', 'ysr_56b', 'ysr_56c', 'ysr_56d', 'ysr_56e',

'ysr_56f', 'ysr_56 $\left.\left.\mathrm{gg}^{\prime}\right)\right]$ \% \% polychoric() \%>\% .'\$rho \%>\% aipha() \%>\% '. \$total

\# Attention_Deficit_Hyperactivity_Problems

Data_set[, c('ysr_04', 'ysr_08', 'ysr_10', 'ysr_41', 'ysr_78', 'ysr_93', 'ysr_104')] \%>\% polychoric() \%>\% .\$rho \%>\% alpha() \%>\%. \$total

\#Oppositiona1_Defiant_Problems

Data_set [, c('ysr_03', 'ysr_22', 'ysr_23', 'ysr_86', 'ysr_95')] \%>\% polychoric() \%>\% . \$rho \%>\% alpha() \%>\% . \$total

\#Conduct_Problems

Data_set [, c('ysr_16', 'ysr_21', 'ysr_26', 'ysr_28', 'ysr_37', 'ysr_39', 'ysr_43', 'ysr_57', 'ysr_67', 'ysr_72', 'ysr_81', 'ysr_82', 'ysr_90', 'ysr_97', 'ysr_101')] \%>\% poiychoric()'\%>\% .\$rho'\%>\% āpha() \%>\% . \$total

\#\#Missing data APQ_YSR

table (is.na(APQ_YSR))

random2 $=\operatorname{rchisq}\left(\operatorname{nrow}\left(A P Q \_Y S R[,-c(1,2)]\right), 14\right)$

fake2 $=1 \mathrm{~m}($ random2 ., data $=$ APQ_YSR $[,-c(1,2)])$

standardizedResidua1s2 $=$ rstudent (fake2)

qqnorm (standardizedResidua1s2)

abline $(0,1)$

\#\#Normality assumtion test

describe (APQ_YSR)

hist (standardizedResiduals2, breaks $=44$ )

describe(standardizedResidua1s2) 
table(is.na (APQ_YSR))

imputed_APQ_YSR $<-$ mice(APQ_YSR, $m=50$, maxit $=50$, method $=$ 'pmm', seed $=$ 4014)

summary (imputed_APQ_YSR)

imputed_APQ_YSR\$imp\$Positive_Parenting

APQ_YSR_Complete <- complete(imputed_APQ_YSR)

which (is.na(APQ_YSR \$Gen) == T)

APQ_YSR_Complete $=$ APQ_YSR_Complete $[-c(947,950)$,

\#\#\#\#\#\#\#\#\#\#\#\#\#\#\#\#\#APQ_YSR Network Estimation\#\#\#\#\#\#\#\#\#\#\#\#\#\#\#\#\#

parenting_practices_children_m_h <- c('a)Parental involvement', 'b)Positive parenting', 'c) Poor monitoring', 'd) Inconsistent discipline',

'e)Corporal punishment', 'f)Affective problems', 'g)Anxiety problems', 'h) Somatic problems',

'k) Conduct Problems')

'i)ADHD problems', 'j)OD Problems',

parenting_practices_Chi1dren_m_h2 <- C("I", "PP", "PM", "ID", "CP",

"Affec_P", "AP", "SP", "ADHDP", "ODP", "Con_P")

Network_APQ_YSR <- estimateNetwork(APQ_YSR_Complete $[,-c(1,2)]$, defau1t = "huge", tuning $=0.5$ )

\# plot and save high resolution graph

tiff("Fig. 3.tif", family = "ArialMT", units $=$ "cm",

width $=24$, height $=16$, pointsize $=12$, res $=500)$

plot(Network_APQ_YSR, 1ayout = "spring", 1abe1s =

parenting_practices_Children_m_h2, groups = parenting_practices_chi1dren_m_h,

color $=c(' \# 58 D 3 F 7$ ', '\#0040FF', '\#9F81F7', '\#A9E2F3', '\#F7819F',

'\#00FF80', '\#01DF01', '\#9AAFE2E', '\#F7FE2E', '\#FAAC58', '\#DC143C'))

dev.off()

\#\#\#\#\#\#\#\#\#\#\#\#\#\#\#\#\#\#APQ_YSR centrality indices computation\#\#\#\#\#\#\#\#\#\#\#\#\#

tiff("Fig. S4.tif", family = "ArialMT", units = "cm", width $=30$, height $=16$, pointsize $=12$, res $=230$ ) 


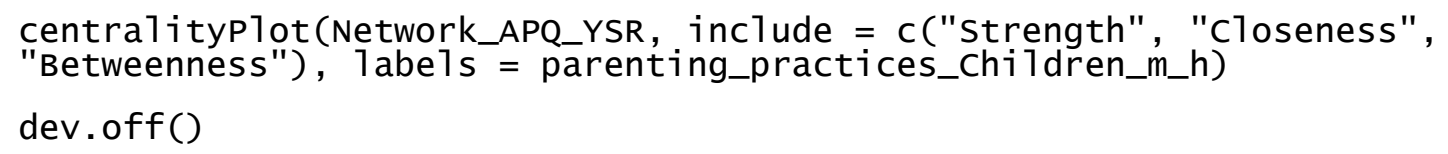




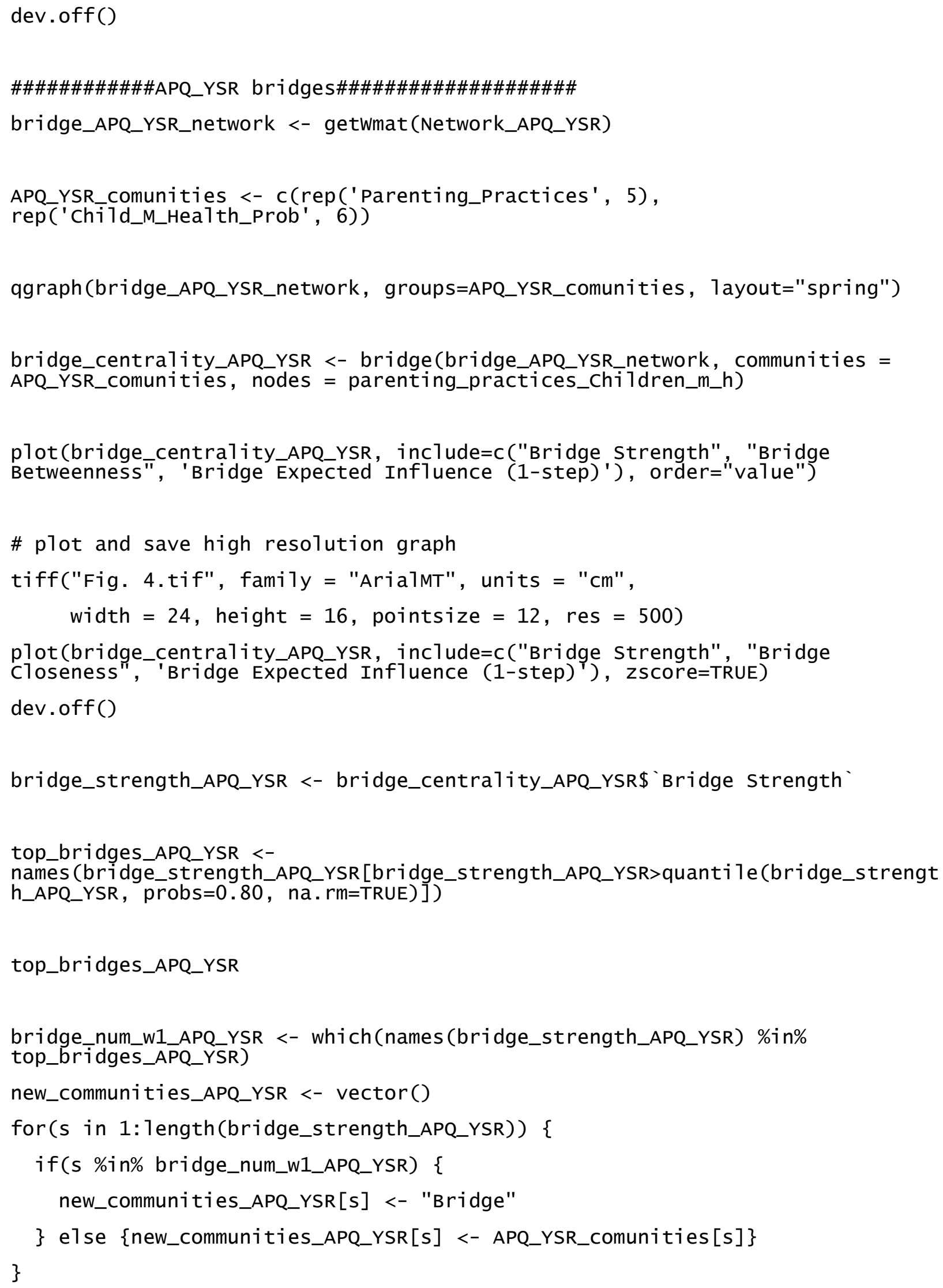


qgraph(bridge_APQ_YSR_network, 1ayout="spring", 1egend = TRUE, groups=new_communities_APQ_YSR, color =

$$
\text { c('\#F78181', '\#40FF00', '\#2E9AFE')) }
$$

\#\#\#\#\#\#\#\#\#\#\#Difference Males-Females Test\#\#\#\#\#\#\#\#\#\#\#\#

APQ_YSR_males <- APQ_YSR_Complete $\%>\%$ filter $(.$, Gen $==1)$

APQ_YSR_females <- APQ_YSR_Complete \%> filter $(.$, Gen $==2)$

Network_males_APQ_YSR <- estimateNetwork(APQ_YSR_ma1es $[,-c(1,2)]$, defau1t = "huge", tuning $=0.5$ )

Network_females_APQ_YSR <- estimatenetwork(APQ_YSR_fema1es [, -c $(1,2)]$, defautt $=$ "huge", tuning $=0.5$ )

plot(Network_females_APQ_YSR, 1ayout = "spring", 1abe1s = parenting_practices_Chi1dren_m_h2, groups = parenting_practices_chi1dren_m_h, color $=c(' \# 58 \mathrm{D} 3 \mathrm{~F} 7$ ', '\#0040FF', '\#9F81F7', '\#A9E2F3', '\#F7819F', '\#00FF80', '\#01DF01', '\#9AFE2E', '\#F7FE2E', '\#FAAC58', '\#F78181'))

plot(Network_ma1es_APQ_YSR, 1ayout = "spring", 1abe1s = parenting_practices_chi1dren_m_h2, groups = parenting_practices_chi1dren_m_h, color = c('\#58D3F7', '\#0040FF', '\#9F81F7', '\#A9E2F3', '\#F7819F', '\#00FF80', '\#01DF01', '\#9AAFE2E', '\#F7FE2E',

'\#FAAC58', '\#F78181'))

Females_males_APQ_YSR_comparison <- NCT(Network_males_APQ_YSR, Network_females_APQ_YSR, it $=1000$, binary.data=FALSE, AND=TRUE, abs=TRUE, test. edges=TRUE, edges $=$ 'a17', make. positive. definite=TRUE, p.adjust.methods = "BH", test.centrality=TRUE, centrality= c("strength", "closeness"), nodes="a17", communities=parenting_practices_children_m_h, usecommunities="a11", verbose = TRUE)

plot(Fema1es_males_APQ_YSR_comparison, what = 'network') 


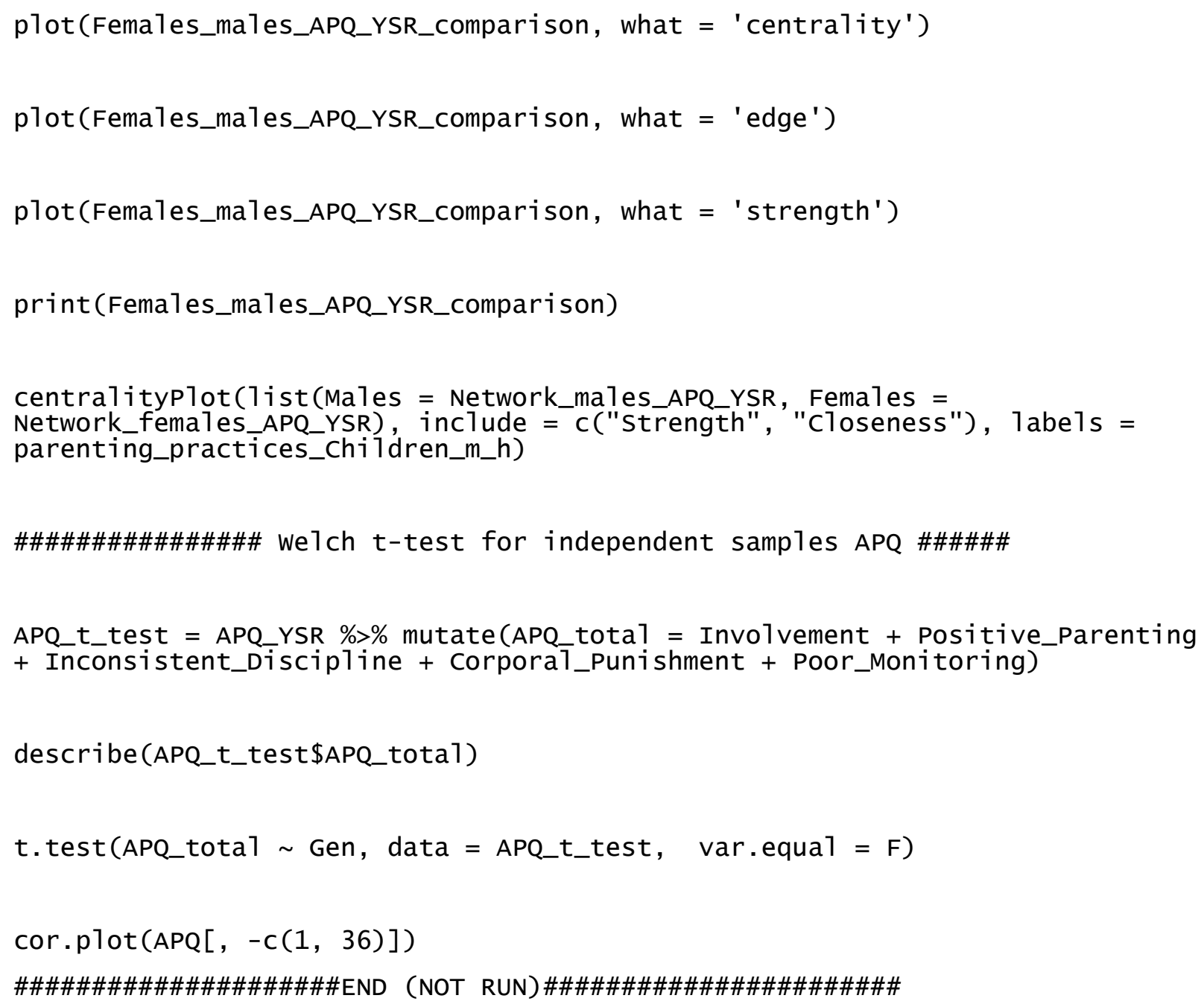

